\title{
Preoperative ultrasonographic findings of internal jugular veins and carotid arteries in kidney transplant recipients
}

\author{
Ji Won Choi, Gaab Soo Kim, Seung Won Lee, Jeong Bo Park, Jeong Jin Lee, \\ and Justin Sangwook Ko \\ Department of Anesthesiology and Pain Medicine, Samsung Medical Center, Sungkyunkwan University School of \\ Medicine, Seoul, Korea
}

\begin{abstract}
Background: Hemodialysis via the internal jugular vein (IJV) has been widely used for patients with end stage renal disease (ESRD) patients, as they have a higher risk of arterial diseases. We investigated the ultrasonographic findings of the IJV and carotid artery (CA) in recipients of kidney transplantation (KT) and identified factors influencing IJV/CA abnormalities.

Methods: We enrolled 120 adult KT recipients. Patients in group A $(n=57)$ had a history of IJV hemodialysis, while those in group B $(n=63)$ were not yet on dialysis or undergoing dialysis methods not involving the IJV. The day before surgery, we evaluated the state of the IJV and CA using ultrasonography. We followed patients with IJV stenosis for six months after KT.

Results: Ultrasonography revealed that four patients (7\%) in group A had IJV abnormalities, while no patients in group $\mathrm{B}$ had abnormalities $(\mathrm{P}=0.118)$. Of the four patients with abnormalities, one with $57.4 \%$ stenosis normalized during follow-up. However, another patient with $90.1 \%$ stenosis progressed to occlusion, while the two patients with total occlusion remained the same. Twenty patients in group A $(n=11)$ and $B(n=9)$ had several CA abnormalities $(P=0.462)$. Upon multivariate analysis with stepwise selection, height and age were significantly correlated with IJV stenosis $(\mathrm{P}=0.043$, odds ratio $=0.9)$ and $\mathrm{CA}$ abnormality $(\mathrm{P}=0.012$, odds ratio $=1.1)$, respectively.

Conclusions: IJV abnormalities (especially with a history of IJV hemodialysis) and CA abnormalities may be present in ESRD patients. Therefore, we recommend ultrasonographic evaluation before catheterization.
\end{abstract}

Key Words: Central venous catheterization, Kidney transplantation, Safety, Ultrasonography.

Corresponding author: Gaab Soo Kim, M.D., Ph.D.

Department of Anesthesiology and Pain Medicine, Samsung Medical Center, Sungkyunkwan University School of Medicine, 81, Irwon-ro, Gangnam-gu, Seoul 06351, Korea

Tel: 82-2-3410-2470, Fax: 82-2-3410-6626

E-mail: gskim@skku.edu

Received: September 2, 2015.

Revised: 1st, January 26, 2016; 2nd, April 5, 2016.

Accepted: April 18, 2016.

Korean J Anesthesiol 2016 August 69(4): 375-381 http://dx.doi.org/10.4097/kjae.2016.69.4.375

\section{Introduction}

The internal jugular vein (IJV) is a preferred site for central venous catheterization for hemodialysis because complications such as stenosis, thrombus, or infection are considered uncommon. However, several papers [1-4] have reported that stenosis of the IJV is associated with hemodialysis and arterial abnormalities, such as wall stiffness, in end stage renal disease (ESRD) patients. Moreover, $26 \%$ of ESRD patients with a history of IJV hemodialysis have anatomical variation of the blood vessels [5].

(c) This is an open-access article distributed under the terms of the Creative Commons Attribution Non-Commercial License (http://creativecommons.org/ licenses/by-nc/4.0/), which permits unrestricted non-commercial use, distribution, and reproduction in any medium, provided the original work is properly cited. 
Thus, the possibility of difficult IJV catheterization and/or accidental injury of the adjacent carotid artery (CA) with thrombus dislodgement may be higher in ESRD patients with a history of IJV hemodialysis.

Moreover, patients with chronic kidney disease (CKD) often present with vascular abnormalities associated with metabolic irregularities related to increased protein degradation products, hormone changes [6,7] or hemodialysis. Metabolic irregularities such as diabetes mellitus (DM) may accelerate atherosclerosis and increase stiffness and thickening of arterial walls [3]. Hemodialysis may also worsen vascular patency, overall or at the site of venous catheterization, and vascular access at the site of previous catheterization may be difficult [8].

In this study, we investigated the state of the IJV and CA in recipients of kidney transplantation using ultrasonography and evaluated the relationship between IJV status and patient history of IJV dialysis. In addition, we attempted to identify factors influencing IJV and CA abnormalities in these patients.

\section{Materials and Methods}

From April 2008 to December 2010, 120 adult recipients, aged 20-65 years, undergoing elective kidney transplantation in our hospital were enrolled in this study. Patients who already had IJV catheters and patients whose IJV catheters were in place for less than one week or used for fewer than three dialysis treatments were excluded from the study. This study was approved by the Institutional Review Board of our institute, and informed consent was obtained from all subjects. This study was registered at http://cris.nih.go.kr (KCT0000198).

The patients were divided into two groups. Group A had a history of IJV hemodialysis, and group B had no history of dialysis or dialysis via other methods, such as peritoneal dialysis or hemodialysis via arteriovenous fistula or the femoral vein. Patency and blood flow of both sides of the IJV and CA were examined at the cricoid level using ultrasonography (iU22 xMATRIX $^{\circledR}$ Philips Medical Systems, Amsterdam, Netherlands) by one of the authors (K.G.S.) and a vascular sonographer at our hospital the day before kidney transplantation. To determine the severity of stenosis as a percentage, we divided the diameter of the most stenotic area in longitudinal view by the diameter of the non-stenotic distal IJV. Six months after kidney transplantation, we re-evaluated the IJV in the same manner in patients with IJV abnormalities.

The day of surgery, after inducing anesthesia and radial artery catheterization, a central venous catheter (Multi-Lumen Central Venous Catheterization Set with ARROWg ${ }^{+}$ard Blue ${ }^{\circledR}$ catheter, Arrow International Inc., PA, USA) was placed through the right internal jugular vein. In case of IJV stenosis or occlusion, central venous catheterization was placed in the patent side. At least $2500 \mathrm{ml}$ of lactated Ringer's solution was infused until reperfusion.

We also determined whether there was atheroma in the wall of the CA, defined as abnormal intima-media thickness on ultrasonography. Additionally, potential confounders of IJV/CA abnormalities such as age, gender, body mass index (BMI), DM, duration of IJV catheterization, and history and duration of dialysis were analyzed.

\section{Statistical analysis}

The null hypothesis of this study was that there is no association in the incidence of IJV stenosis between groups A and B. The alternative hypothesis of this study was that there is an association in the incidence of IJV stenosis between the two groups.

Statistical analyses were carried out using the SAS 9.4 system (SAS Institute, Cary, NC, USA). Differences between the two groups in patient characteristics and relationships among age, gender, BMI, DM, duration of IJV catheterization and IJV stenosis were analyzed using two sample t-tests and Wilcoxon rank sum tests for continuous variables, or Chi-square test and Fisher's exact tests for categorical variables. IJV stenosis and CA abnormalities in relation to several factors were assessed via multivariate logistic regression with stepwise selection. In multivariate logistic regression with stepwise selection, the $\mathrm{P}$ value for entry and stay was 0.1 . P values $<0.05$ were considered statistically significant.

\section{Results}

A total of 120 patients were enrolled. Group A included 57 patients and group $B$ included 63 patients. Thirty-two patients in group B had no history of dialysis and the remaining 31 patients

Table 1. Patient Characteristics and Clinical Data

\begin{tabular}{lccc}
\hline & $\begin{array}{c}\text { Group A } \\
(\mathrm{n}=57)\end{array}$ & $\begin{array}{c}\text { Group B } \\
(\mathrm{n}=63)\end{array}$ & P value \\
\hline Age $(\mathrm{yr})$ & $44.8 \pm 10.6$ & $43.0 \pm 10.8$ & 0.693 \\
& $(19-65)$ & $(17-65)$ & \\
Gender $(\mathrm{M} / \mathrm{F})$ & $29 / 28$ & $39 / 24$ & 0.223 \\
Height $(\mathrm{cm})$ & $164.2 \pm 10.1$ & $165.7 \pm 8.7$ & 0.482 \\
Weight $(\mathrm{kg})$ & $58.5 \pm 12.3$ & $60.1 \pm 11.4$ & 0.467 \\
BMI $\left(\mathrm{kg} / \mathrm{m}^{2}\right)$ & $21.7 \pm 3.6$ & $22.0 \pm 3.0$ & 0.703 \\
DM (n) & $11(19.3)$ & $11(17.5)$ & 0.795 \\
IJV stenosis (n) & $4(7.02)$ & 0 & $0.118^{*}$ \\
CA abnormalities $(\mathrm{n})$ & $11(19.3)$ & $9(14.3)$ & 0.462 \\
\hline
\end{tabular}

Data are mean \pm SD (range) or number of patients (percentage). Group A: patients with a history of IJV haemodialysis, Group B: patients without a history of IJV hemodialysis, BMI: body mass index, DM: diabetes mellitus, IJV: internal jugular vein, CA: carotid artery. ${ }^{*}$ The value was analysed by Firth's penalized likelihood approach. 

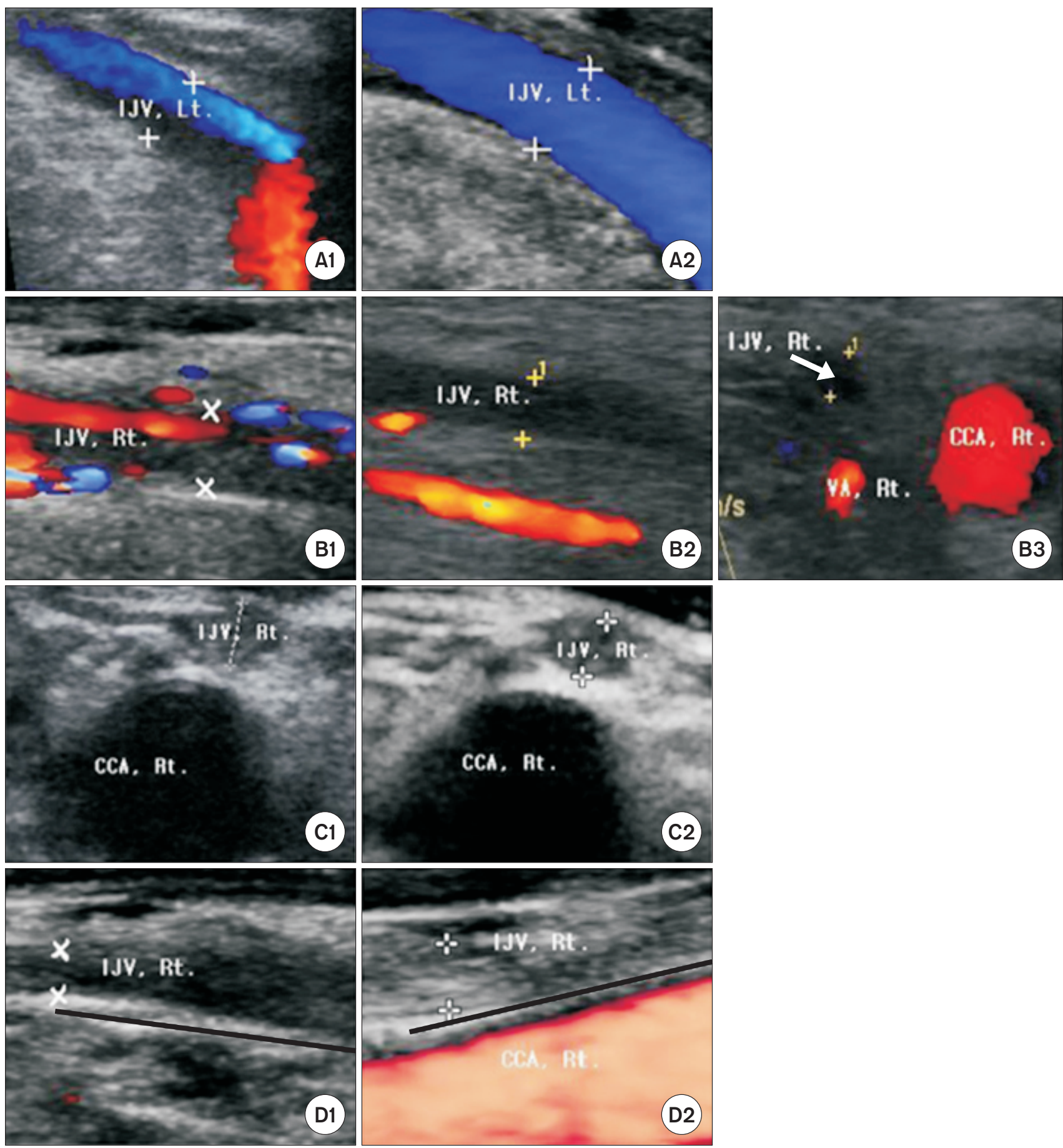

Fig. 1. Longitudinal (A1, A2, B1, B2, D1, D2), and cross-sectional (B3, C1 ,C2) Doppler images in the four patients who showed partial or total obstruction of the internal jugular vein. The partial obstruction of the left IJV (A1) restored to original patency six months later (A2). Near total obstruction of the right internal jugular vein (B1) progressed to total obstruction six month later (B2, B-3). The lumen was filled with thrombus. There was no flow in Doppler images of patients (C1) and (D1). These images show two cases of total stenosis of the right internal jugular vein in the proximal $3 \mathrm{~cm}$ portion (This portion is marked by black line in D1 and D2). Six months later, both cases maintained the same state (C2) and (D2). Each lumen was filled with thrombus. IJV: internal jugular vein, CCA: common carotid artery, VA: vertebral artery, Rt: right, Lt: left. ' + ' is the marker to identify blood vessel wall. 

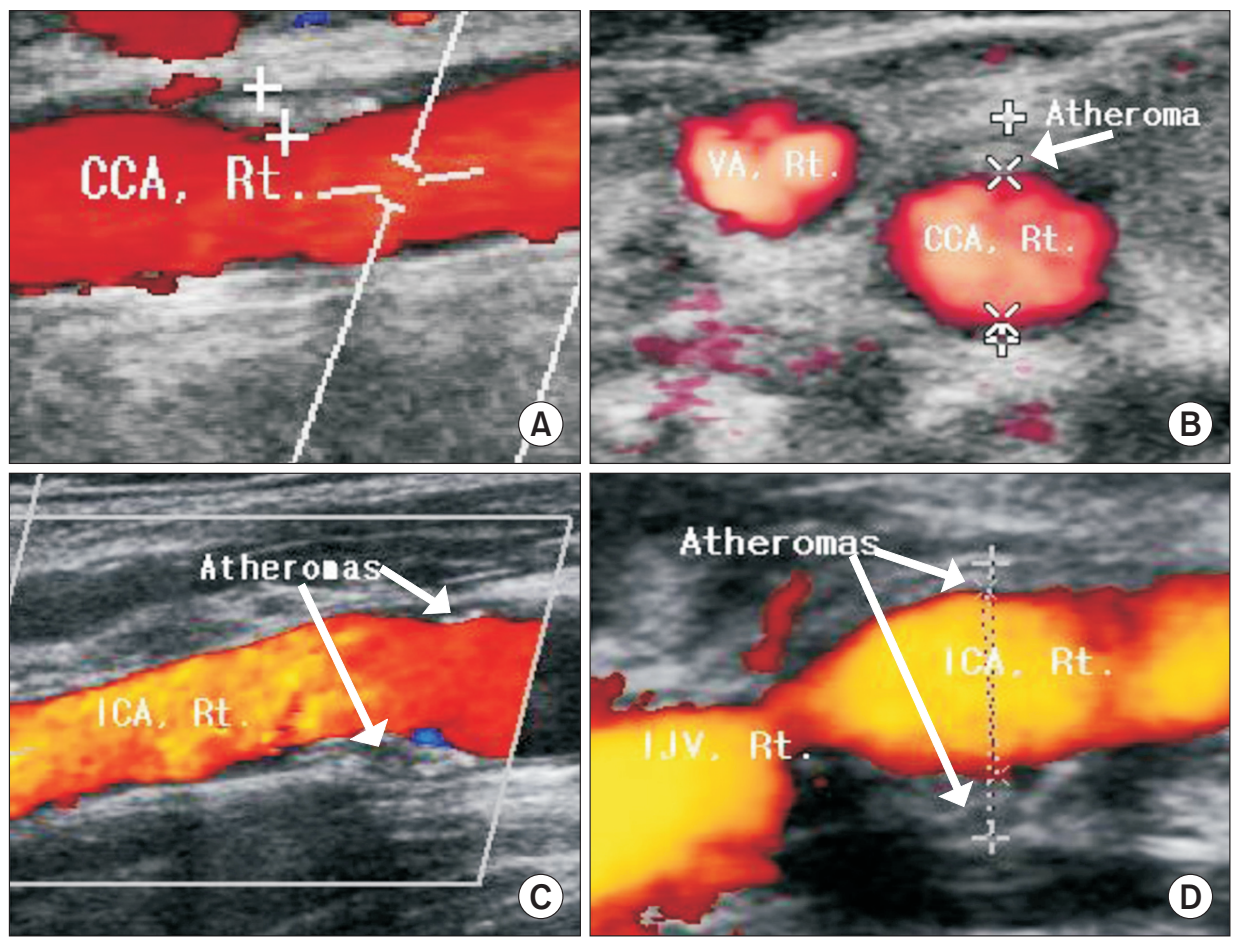

Fig. 2. These color and power Doppler images show two cases of wall thickening in the common carotid and internal carotid arteries $(\mathrm{A}, \mathrm{C})$ on longitudinal view, with atheromas in their lumens $(B$, D) on cross-sectional view. $\mathrm{A} / \mathrm{B}$ and $\mathrm{C} / \mathrm{D}$ are the same patients, respectively. CCA: common carotid artery, VA: vertebral artery, ICA: internal carotid artery, IJV: internal jugular vein, Rt: right.

had a history of dialysis via a method other than IJV. Patient characteristics, including CA abnormality, were comparable between the two groups (Table 1). In group A, 52 patients were catheterized in the right side of the IJV and 5 patients in both sides. The duration of IJV catheterization was $5.86 \pm 4.74$ (mean $\pm \mathrm{SD}$ ) weeks, and the interval between IJV catheter removal and ultrasonographic evaluation was $47.7 \pm 46.8$ months.

Ultrasonography revealed that four (7\%) patients in group A had IJV abnormalities. No patients in group B had abnormalities. Since there were zero cells in a $2 \times 2$ table on Fisher's exact test, we performed Firth's penalized likelihood approach in a logistic regression model to compute the odds ratio. On logistic regression analysis, the odds of IJV abnormality in group A were 10.7 times the odds in group $\mathrm{B}(\mathrm{P}=0.118,95 \% \mathrm{CI}=0.6-207.8)$. Four patients from group A showed IJV abnormalities, including two cases of stenosis and two occlusions. The stenosis or occlusion sites (right or left) of the four patients were on the same side as previous catheterization. The severity of stenosis was $57.4 \%$ in one patient and $90.1 \%$ in the other. In both patients with total occlusion, the occlusions were $3 \mathrm{~cm}$ long and just proximal to and around the hemodialysis catheter insertion site (Figs. 1A-1D). The four patients with IJV abnormalities had undergone $16,4,8$ and 8 weeks of catheterization, respectively, and the interval between the removal of the hemodialysis catheter and ultrasonographic examination was 108, 36, 23 and 39 months, respectively. Patients in group B did not show any ste- nosis or occlusion of the IJV.

At the six-month follow-up visit of the four IJV abnormality patients (the two stenosis and two occlusion cases), only the patient with $57.4 \%$ stenosis showed normalized patency and blood flow. The stenotic lesion progressed to occlusion in the patient with $90.1 \%$ stenosis. Both occlusion cases remained the same (Figs. 1. A2-D2 and B3).

Twenty patients total (16.7\%), eleven in group A and nine in group B, had several CA abnormalities, including wall thickening estimated with atheroma resulting in stenosis (Fig. 2). Among them, ten patients had abnormalities on both sides, six had left side abnormalities and four had right side abnormalities.

When we subdivided the patients into two groups according to the presence of IJV stenosis or CA abnormalities, the odds ratio of height for IJV stenosis was 0.9 (95\% CI 0.8-1.0, P = 0.043 ) (Table 2) and the odds ratio of age and DM for CA abnormalities were 1.1 (95\% CI 1.1-1.2, $\mathrm{P}=0.001)$ and $3.1(95 \%$ CI 1.1-8.9, P = 0.041) (Table 3), respectively, in univariate analysis. On multivariate analysis with stepwise selection, height was significantly correlated with IJV stenosis $(\mathrm{P}=0.043$, odds ratio $=0.9$ ) and only age was significantly correlated with CA abnormality $(\mathrm{P}=0.012$, odds ratio $=1.1)$. To validate the regression model, we performed Pearson Goodness-of-Fit test for each multivariate regression model. We concluded that the models fit the data well $(\mathrm{P}=0.980$ and $\mathrm{P}=0.615)$. 
Table 2. Factors Potentially Influencing Internal Jugular Vein Stenosis in Univariate Analysis

\begin{tabular}{lcccc}
\hline & $\begin{array}{c}\text { IVJ stenosis }(-) \\
(\mathrm{n}=116)\end{array}$ & $\begin{array}{c}\text { IVJ stenosis }(+) \\
(\mathrm{n}=4)\end{array}$ & $\beta$ (95\% confidence interval) & P value \\
\hline Age $(\mathrm{yr})$ & $43.7 \pm 11.1$ & $53.3 \pm 1.9$ & $0.10(-0.02,0.22)$ & 0.103 \\
Sex $(\mathrm{M} / \mathrm{F})$ & $68 / 48$ & $0 / 4$ & $-2.54(-5.51,0.43)$ & 0.093 \\
Height $(\mathrm{cm})$ & $165.2 \pm 9.4$ & $154.9 \pm 5.3$ & $-0.13(-0.25,0.004)$ & 0.043 \\
Weight $(\mathrm{kg})$ & $59.5 \pm 11.8$ & $55.4 \pm 12.6$ & $-0.04(-0.14,0.07)$ & 0.494 \\
BMI $\left(\mathrm{kg} / \mathrm{m}^{2}\right)$ & $21.7 \pm 3.3$ & $20.5 \pm 5.3$ & $0.10(-0.16,0.36)$ & 0.455 \\
DM $(\mathrm{n}, \%)$ & $21(18)$ & $1(25)$ & $0.41(-1.90,2.72)$ & 0.728 \\
Duration of dialysis (week) & $53.0 \pm 7.1$ & $16,4,8,8^{*}$ & $0.01(-0.06,0.09)$ & 0.726 \\
\hline
\end{tabular}

Data are mean \pm SD or number of patients (percentage). BMI: body mass index, DM: diabetes mellitus, IVJ: internal jugular vein. *Actual values for duration of dialysis of four patients.

Table 3. Factors Potentially Influencing Carotid Artery Abnormalities in Univariate Analysis

\begin{tabular}{|c|c|c|c|c|}
\hline & $\begin{array}{c}\text { CA abnormality }(-) \\
\quad(\mathrm{n}=100)\end{array}$ & $\begin{array}{c}\text { CA abnormality }(+) \\
(\mathrm{n}=20)\end{array}$ & $\beta(95 \%$ confidence interval $)$ & $P$ value \\
\hline Age (yr) & $42.2 \pm 10.8$ & $53.0 \pm 7.7$ & $0.11(0.04,0.17)$ & 0.001 \\
\hline $\operatorname{Sex}(M / F)$ & $57 / 43$ & $43 / 9$ & $0.16(-0.81,1.14)$ & 0.742 \\
\hline Height $(\mathrm{cm})$ & $165.4 \pm 9.2$ & $162.3 \pm 10.5$ & $-0.03(-0.1,0.02)$ & 0.281 \\
\hline Weight (kg) & $59.6 \pm 11.8$ & $58.0 \pm 12.2$ & $0.01(-0.03,0.05)$ & 0.562 \\
\hline $\operatorname{BMI}\left(\mathrm{kg} / \mathrm{m}^{2}\right)$ & $21.7 \pm 3.4$ & $21.9 \pm 3.2$ & $0.11(-0.03,0.24)$ & 0.125 \\
\hline $\mathrm{DM}(\mathrm{n}, \%)$ & $15(15)$ & $7(35)$ & $1.12(0.05,2.19)$ & 0.041 \\
\hline Hypertension (n, \%) & $61(61)$ & $16(80)$ & $0.94(-0.23,2.11)$ & 0.115 \\
\hline Duration of dialysis (week) & $150(48.0-330.2)$ & $184(102.1-408.3)$ & $0.02(-0.03,0.08)$ & 0.431 \\
\hline IJV stenosis (n, \%) & $2(2)$ & $2(10)$ & $1.70(-0.33,3.72)$ & 0.101 \\
\hline
\end{tabular}

Data are mean \pm SD or number of patients (percentage) or median (Q1-Q3). BMI: body mass index, CA: carotid artery, DM: diabetes mellitus, IVJ: internal jugular vein.

\section{Discussion}

ESRD patients may experience sudden deterioration leading to emergency room visits and the need for kidney transplantation. In many cases, a central venous catheter must be inserted along with the hemodialysis catheter. The IJV is considered the best and safest route for temporary vascular access or central venous route in ESRD patients who need long- or short-term dialysis for a variety of conditions. Therefore, it is necessary to confirm the state of the IJV before catheterization to prevent complications associated with a difficult procedure, especially if there is a history of hemodialysis via the IJV. Considering anatomical variations of the IJV, use of ultrasonography during IJV catheterization is recommended.

Several studies have reported the utility of ultrasonography for viewing blood vessels. For example, ultrasonography-guided catheterization of the IJV for acute hemodialysis or in critical care patients has been reported [9-12]. In this study, we used ultrasonography to evaluate the IJV and CA in ESRD patients waiting for elective kidney transplantation. We identified abnormalities and followed cases of IJV stenosis.

Fant et al. [13] described patients with strictures several weeks to months after catheter removal, indicating that both stricture and thrombosis are possible in the long term after temporary dialysis has ceased. In this study, patients with IJV abnormalities underwent, on average, $9.0 \pm 5.03$ weeks of catheterization, and the interval between the removal of the hemodialysis catheter and ultrasonographic examination was $51.5 \pm$ 38.3 months.

Interestingly, the case with $57.4 \%$ stenosis resolved six months after kidney transplantation. However, the case with 90.1\% stenosis progressed to occlusion, and two other cases of occlusion remained the same. Based on these results, we infer that there is a relationship between stenosis severity and the future condition of the blood vessels. In cases of partial stenosis, there may be the possibility of recovery. If the patient has near or total occlusion, the lesion may not recover or may progress further. Although it is difficult to generalize based on such a small number of cases, the potential for stenotic progression should be taken into account when IJV hemodialysis catheters are placed.

The incidence of stenosis has been reported to be $0-10 \%$ in the IJV [4,14], consistent with the 7.02\% (4/57) incidence observed in this study. Numerous studies have shown stenosis or occlusion of the subclavian vein or IJV secondary to previous 
catheterization $[2,4,15,16]$. Intimal trauma with endothelial disruption at the puncture site is a frequent cause of stricture. Stiffness of the catheter can encourage stenosis [17]. Several other factors, including age, gender, number of punctures, duration of catheterization and number of dialysis sessions are thought to be related to stenosis, but these have not been confirmed. Complications such as poor flow or infection favor thrombosis and perhaps stenosis [4].

In this study, height was significantly correlated with IJV stenosis. All four patients with IJV stenosis were short in stature. In general, hemodialysis catheters have an internal diameter unadjusted to the catheter length. If catheter diameter is so large as to fill the vein too tightly, it could predispose for damage of the vein wall, thrombosis, and stenosis. Twardowski and Seger [18] reported that the lengths of the veins were correlated with the body anthropometric measurements, such as height, weight, body surface area, and bi-acromion span. However, further studies are needed to support our results, which were derived from a small sample size.

The spectrum of arterial alterations in ESRD is broad, including occlusive lesions caused by atheromatous plaques and arterial remodeling accompanying the growing hemodynamic burden in conjunction with the natural aging process [19]. In this study, arterial wall thickness or plaque due to calcification or atheroma was observed in $16.7 \%$ of patients. This incidence is much higher than the $5.5 \%$ reported for individuals undergoing routine heath examination [20]. ESRD patients show accelerated rates of atherosclerosis and microangiopathic changes, and calcification in the CA and coronary arteries of young patients on hemodialysis has been reported [21].

Some studies have reported that artery punctures occur with a frequency of $1.39-1.72 \%$ despite the use of ultrasound-guided techniques $[22,23]$. If the CA is punctured, there may be potential for disruption of plaque, and the resulting embolism may cause brain infarction. Decreased carotid flow can be aggravated if the neck is compressed to prevent hematoma. In some cases, it has been reported that a carotid-jugular arteriovenous fistula or resulting cerebral infarct have occurred because of a thrombo- embolism from an arterial atherosclerotic plaque secondary to catheterization for hemodialysis [24,25].

In general, ESRD patients have a greater number of CA abnormalities, especially those that are dialysis-dependent [1]. In this study, old age also increased the frequency of CA abnormalities, consistent with the results of recent large-scale research [26]. Therefore, regardless of whether an ultrasound-guided technique is used, physicians should be mindful of the increased risk of atheroma separation when sticking an artery with a needle and be especially careful to not injure the CA at the time of IJV catheterization in elderly ESRD patients.

This study has several limitations. First, central vein catheterization is related to stenosis. In the present study, we paid special attention to patients with IJV stenosis and followed them. By following patients with IJV stenosis for several months after kidney transplantation, we attempted to examine long-term vessel change. Second, we could not calculate exact sample size. Because the sample size was small, there would not have been significant results in the odds ratio of IJV abnormality between the two groups. For the same reason, we were unable to definitively determine risk factors affecting IJV stenosis because of our small sample size. Future studies looking at a broad population will be necessary to generalize our results. Lastly, we investigated only a few factors affecting carotid artery abnormalities. Other factors affecting arteriopathy, such as smoking, cholesterol level, and kidney function, could not be analyzed. In future studies with larger populations, we will analyze several more factors affecting CA abnormalities.

In conclusion, IJV abnormality may occur in ESRD patients with a history of IJV hemodialysis, which may progress or remain after kidney transplantation. ESRD patients also have higher rates of CA abnormalities. Thus, it is helpful to evaluate the state of the IJV and CA before IJV catheterization.

\section{ORCID}

Ji Won Choi, http://orcid.org/0000-0001-7403-2863

\section{References}

1. Brzosko S, Lebkowska U, Malyszko J, Hryszko T, Krauze-Brzosko K, Mysliwiec M. Intima media thickness of common carotid arteries is associated with traditional risk factors and presence of ischaemic heart disease in hemodialysis patients. Physiol Res 2005; 54: 497-504.

2. Campistol JM, Almirall J, Rello J, Revert L. Jugular vein cannulation for hemodialysis access. Nephron 1988; 50: 391-2.

3. Ritz E. Atherosclerosis in dialyzed patients. Blood Purif 2004; 22: 28-37.

4. Schillinger F, Schillinger D, Montagnac R, Milcent T. Post catheterisation vein stenosis in haemodialysis: comparative angiographic study of 50 subclavian and 50 internal jugular accesses. Nephrol Dial Transplant 1991; 6: 722-4.

5. Lin BS, Kong CW, Tarng DC, Huang TP, Tang GJ. Anatomical variation of the internal jugular vein and its impact on temporary haemodialysis vascular access: an ultrasonographic survey in uraemic patients. Nephrol Dial Transplant 1998; 13: 134-8. 
6. Bahous SA, Stephan A, Blacher J, Safar ME. Aortic stiffness, living donors, and renal transplantation. Hypertension 2006; 47: 216-21.

7. Covic A, Haydar AA, Bhamra-Ariza P, Gusbeth-Tatomir P, Goldsmith DJ. Aortic pulse wave velocity and arterial wave reflections predict the extent and severity of coronary artery disease in chronic kidney disease patients. J Nephrol 2005; 18: 388-96.

8. Vanherweghem JL, Cabolet P, Dhaene M, Goldman M, Stolear JC, Sabot JP, et al. Complications related to subclavian catheters for hemodialysis. Report and review. Am J Nephrol 1986; 6: 339-45.

9. Farrell J, Gellens M. Ultrasound-guided cannulation versus the landmark-guided technique for acute haemodialysis access. Nephrol Dial Transplant 1997; 12: 1234-7.

10. Slama M, Novara A, Safavian A, Ossart M, Safar M, Fagon JY. Improvement of internal jugular vein cannulation using an ultrasound-guided technique. Intensive Care Med 1997; 23: 916-9.

11. Karakitsos D, Labropoulos N, De Groot E, Patrianakos AP, Kouraklis G, Poularas J, et al. Real-time ultrasound-guided catheterisation of the internal jugular vein: a prospective comparison with the landmark technique in critical care patients. Crit Care 2006; 10 : R162.

12. Turker G, Kaya FN, Gurbet A, Aksu H, Erdogan C, Atlas A. Internal jugular vein cannulation: an ultrasound-guided technique versus a landmark-guided technique. Clinics (Sao Paulo) 2009; 64: 989-92.

13. Fant GF, Dennis VW, Quarles LD. Late vascular complications of the subclavian dialysis catheter. Am J Kidney Dis 1986; 7: 225-8.

14. Cimochowski GE, Worley E, Rutherford WE, Sartain J, Blondin J, Harter H. Superiority of the internal jugular over the subclavian access for temporary dialysis. Nephron 1990; 54: 154-61.

15. Ponz E, Campistol Plana JM, Almirall J, Sala X, Revert L. Mechanism of hemodialysis-associated subclavian vein stenosis. Nephron 1990; 56: $227-8$

16. Schwab SJ, Quarles LD, Middleton JP, Cohan RH, Saeed M, Dennis VW. Hemodialysis-associated subclavian vein stenosis. Kidney Int 1988; 33: 1156-9.

17. Bozzetti F, Scarpa D, Terno G, Scotti A, Ammatuna M, Bonalumi MG, et al. Subclavian venous thrombosis due to indwelling catheters: a prospective study on 52 patients. JPEN J Parenter Enteral Nutr 1983; 7: 560-2.

18. Twardowski ZJ, Seger RM. Dimensions of central venous structures in humans measured in vivo using magnetic resonance imaging: implications for central-vein catheter dimensions. Int J Artif Organs 2002; 25: 107-23.

19. London GM, Guerin AP, Marchais SJ, Pannier B, Safar ME, Day M, et al. Cardiac and arterial interactions in end-stage renal disease. Kidney Int 1996; 50: 600-8.

20. Lee KB, Kim DI, Cho JH, Choi YH, Kim SW, Moon JY, et al. Prevalence and risk factors of asymptomatic carotid stenosis in healthy Korean population. J Korean Surg Soc 2004; 66: 415-9.

21. Shoji T, Emoto M, Tabata T, Kimoto E, Shinohara K, Maekawa K, et al. Advanced atherosclerosis in predialysis patients with chronic renal failure. Kidney Int 2002; 61: 2187-92.

22. Denys BG, Uretsky BF, Reddy PS. Ultrasound-assisted cannulation of the internal jugular vein. A prospective comparison to the external landmark-guided technique. Circulation 1993; 87: 1557-62.

23. Troianos CA, Jobes DR, Ellison N. Ultrasound-guided cannulation of the internal jugular vein. A prospective, randomized study. Anesth Analg 1991; 72: 823-6.

24. Bahcebasi S, Kocyigit I, Akyol L, Unal A, Sipahioğlu MH, Oymak O, et al. Carotid-jugular arteriovenous fistula and cerebrovascular infarct: a case report of an iatrogenic complication following internal jugular vein catheterization. Hemodial Int 2011; 15: 284-7.

25. el-Shahawy MA, Khilnani H. Carotid-jugular arteriovenous fistula: a complication of temporary hemodialysis catheter. Am J Nephrol 1995; 15: 332-6.

26. Asakawa T, Hayashi T, Tanaka Y, Joki N, Hase H. Changes over the last decade in carotid atherosclerosis in patients with end-stage kidney disease. Atherosclerosis 2015; 240: 535-43. 